\title{
Les différents scénarios du changement climatique
}

\author{
Michel DÉQUÉ \\ Météo-France, \\ Centre national de recherches météorologiques, \\ CNRS/GAME, \\ 42 av Coriolis \\ 31057 Toulouse, France \\ $<$ deque@meteo.fr>
}

\begin{abstract}
The assumption of a climate change along the 21st century is now widely accepted : the world population and consumption growth will lead to an increase in greenhouse effect. In order to explore possible evolutions in agreement with the laws of physics, numerical modeling is the most appropriate tool. ARPEGE-Climate is the atmospheric model of the French meteorological service. It has been run over the $21^{\text {st }}$ century to simulate possible evolutions under three hypotheses of increase in greenhouse gas and aerosol concentration. This model covers the globe with a rather high resolution over southern Europe $(50 \mathrm{~km})$. The results show a larger warming in summer than in winter and a spatially variable response for precipitation.
\end{abstract}

Key words: climate change, numerical modelling, greenhouse effect, France, scenario, atmosphere

couplés océan-atmosphère pour calculer l'évolution des températures de l'océan et de l'étendue de banquise. Le maillon suivant de la chaîne est le modèle régional de climat, dont les résultats sont utilisés par les modèles d'impact.

Le modèle ARPEGE est le modèle atmosphérique de Météo-France, servant à la fois aux prévisions météorologiques, aux simulations couplées océan-atmosphère, et aux scénarios climatiques régionaux. Les trois scénarios sont étudiés ici sont, par ordre d'intensité décroissante, A2, A1B et B1. De 1950 à 2000, le modèle utilise les forçages radiatifs observés. Jusqu'en 2050 les trois scénarios sont semblables en terme de forçage radiatif et les différences entre les climats simulés sont dus pour l'essentiel à la variabilité naturelle du système climatique. Nous nous concentrerons ici sur deux périodes : 1961-1990 pour le climat de référence et 2071-2100 pour la réponse anthropique.

\section{Description du modèle}

L'utilisation d'un modèle global à haute résolution [2] est la méthode la plus simple et naturelle pour étudier le climat régional, mais c'est aussi la plus chère en ressources de calcul. Aussi, des modèles à aire limitée emboîtés dans des modèles globaux [3] sont le plus souvent utilisés. Les méthodes d'adaptation statistique [4] offrent une autre solution moins onéreuse mais plus difficile à justifier. L'étude présente est effectuée avec la première catégorie de modèle, qui offre des simulations cohérentes sur le globe en s'affranchissant des soucis numériques de la deuxième catégorie et des limitations liées à l'empirisme de la troisième catégorie. Pour réduire le coût de calcul, la résolution n'est élevée que sur une région centrée sur la Méditerranée [5]. Les températures de surface de la mer (TSM) sont fournies par des simulations du modèle à plus basse résolution de Météo-France (maille 300 km), couplé à un modèle d'océan de résolution similaire. Le modèle d'ARPEGE-Climat a été utilisé dans sa version 4 pour la présente étude. Cette version est sommairement décrite dans [6]. On trouvera la description complète sur le site : http://www.cnrm.meteo.fr/gmgec/site_engl/ index_en.html

Par rapport à la version utilisée pour la prévision opérationnelle à courte échéance (un à cinq jours), sa résolution horizontale est un peu moins fine (50 km contre $15 \mathrm{~km}$ sur la France). Par contre, on essaie de prendre en compte plus de phénomènes physiques (par exemple le vieillissement de la neige) car l'impact sur I'atmosphère à long terme n'est pas négligeable contrairement à une prévision à 4 jours. Le calcul du bilan radiatif est modifié par cinq types d'aérosols (désertiques, carbonés, marins, sulfatés et organiques), par cinq gaz à effet de serre (dioxyde de carbone, oxyde nitreux, méthane, chloro-fluoro-carbones et ozone) et par l'eau (vapeur et nuages). Ce modèle gère aussi l'évolution de la surface continentale par le modèle ISBA. En particulier, une variable du modèle représente la quantité d'eau liquide du sol à disposition des racines pour le calcul de l'évapo-transpiration.

\section{Description des expériences}

Les forçages radiatifs (concentrations en gaz à effet de serre et en aérosols sulfatés) sont ceux 


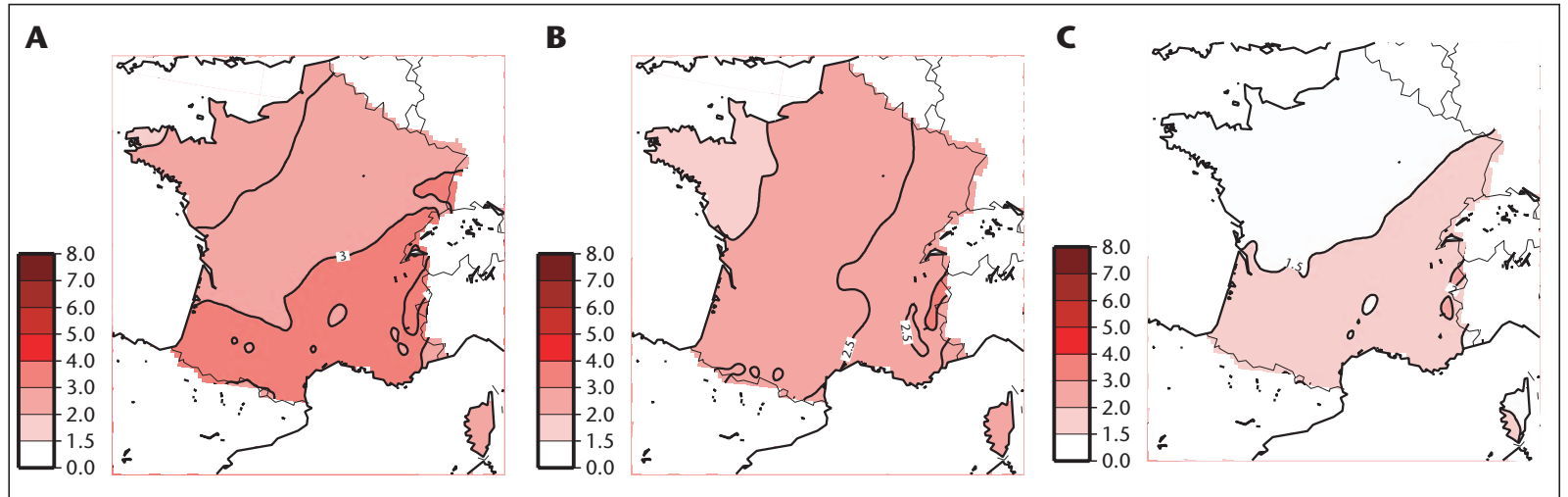

Figure 1. Différence de température hivernale entre 2071-2100 et 1961-1990 pour les scénarios $A 2(a), A 1 B(b)$ et $B 1$ (c); écart des isothermes $0,5^{\circ} \mathrm{C}$.

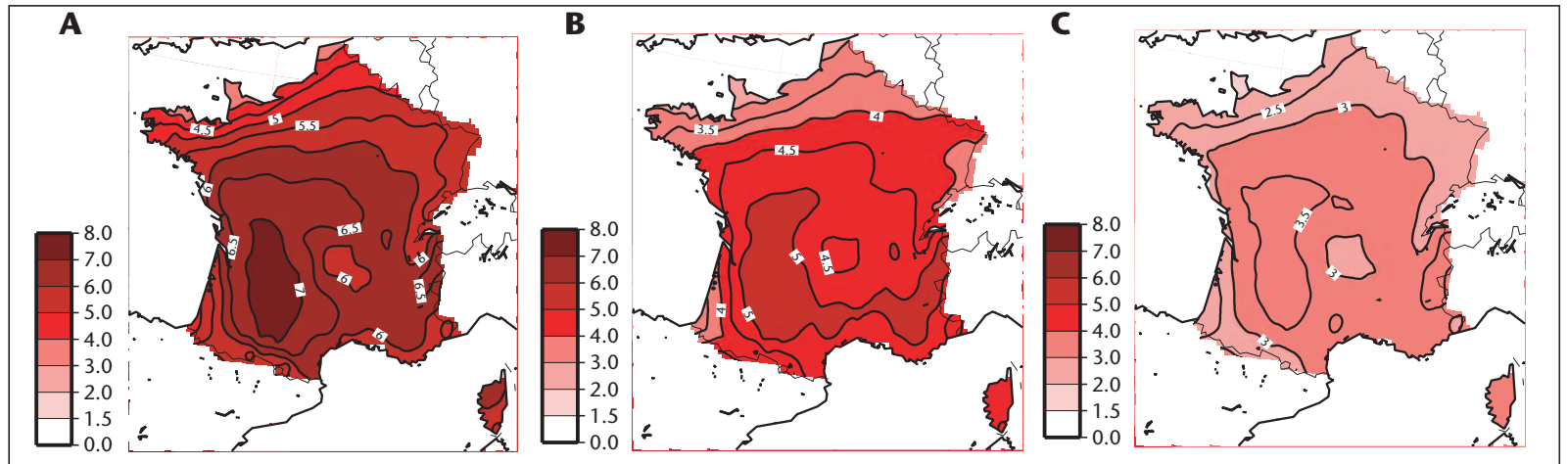

Figure 2. Comme la figure 1 pour l'été.

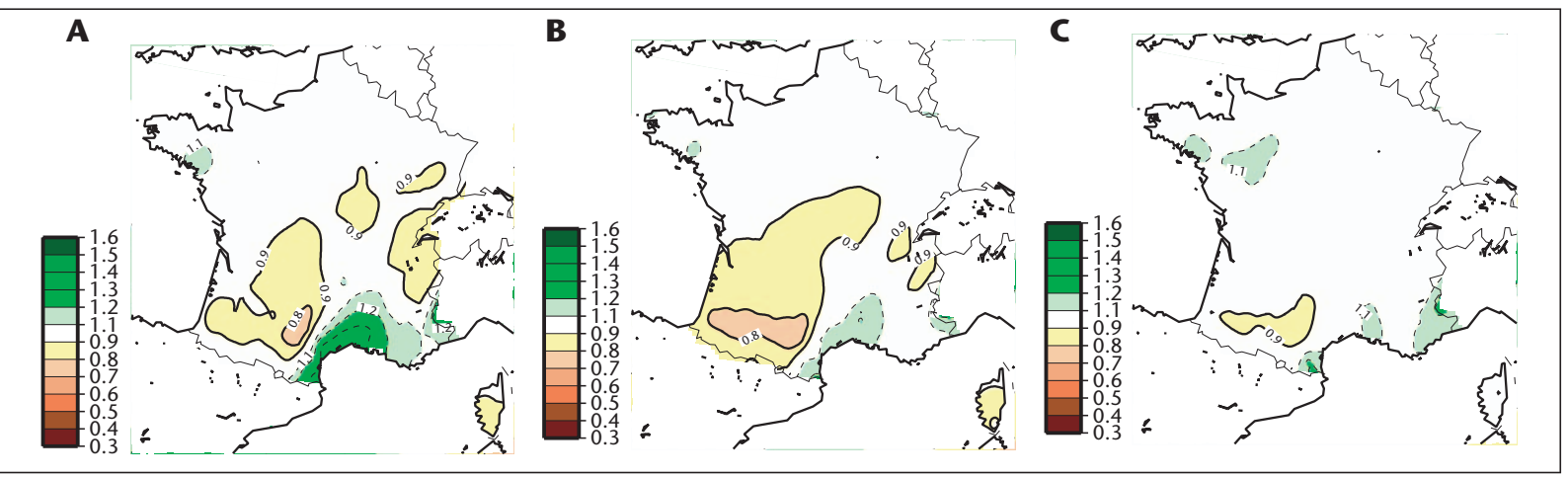

Figure 3. Rapport des précipitations hivernales entre 2071-2100 et 1961-1990 pour les scénarios A2 (a), A1B (b) et B1 (c); écart des isolignes 0,1; l'isoligne 1 n'est pas représentée et les isolignes supérieures à 1 sont présentées en tireté.

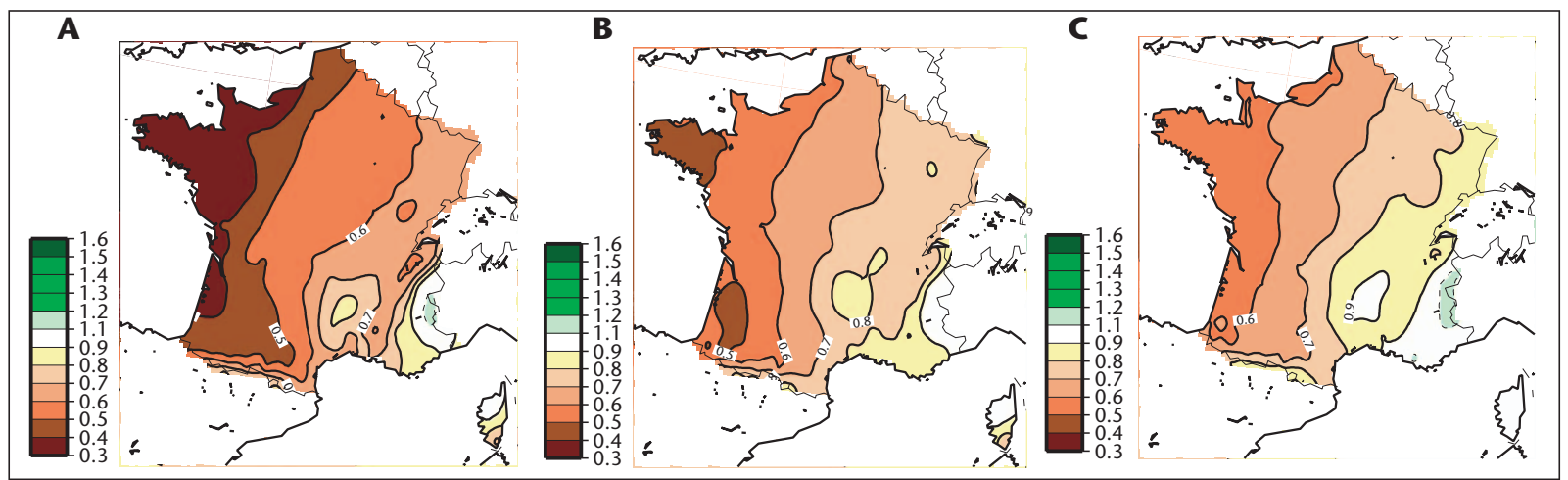

Figure 4. Comme la figure 3 pour l'été. 
des scénarios étudiés par le GIEC dans son $4 \mathrm{e}$ rapport [1]. Le scénario B1 propose une croissance quasi linéaire de la concentration en gaz à effet de serre, conduisant à un doublement en 2100 par rapport à la concentration préindustrielle. Le scénario A2 est plus pessimiste et propose une croissance de type exponentiel, avec triplement en 2100 par rapport à la valeur préindustrielle. Le scénario A1B se situe entre les deux. Les températures de surface de la mer (TSM) sont précalculées, car le modèle $d^{\prime}$ atmosphère $n^{\prime}$ est pas couplé à un modèle d'océan. Elles proviennent des moyennes mensuelles des simulations couplées. Le modèle global d'océan est OPA8.0 développé à l'Institut Pierre Simon Laplace (IPSL) et le modèle de banquise GELATO développé à Météo-France. Un biais pour chaque point et chaque mois calendaire est calculé pour les TSM de 1958 à 2000. Ce biais est ensuite retranché aux simulations régionales, qui couvrent la période 1951-2100 dans le cas du scénario médian A1B et les sous-périodes 2021-2050 et 2071-2100 pour les scénarios extrêmes A2 et B1. L'utilisation des TSM brutes par le modèle régional conduirait à des erreurs systématiques par rapport au climat observé assez importantes. Certes, le modèle régional ainsi piloté comporte encore des erreurs systématiques sur la période 1951-2000, mais en faisant l'hypothèse, communément admise et incontournable, que ces erreurs ne changent pas énormément au cours du XXI ${ }^{e}$ siècle, on pourra s'en affranchir dans la section 4.

Ces scénarios régionaux ont été utilisés dans les projets européens ( $6^{\mathrm{e}}$ Programme cadre de recherche et développement) ENSEMBLES et CECILIA pour piloter des modèles à aire limitée de résolution plus fine. Ils ont aussi été utilisés dans le projet ANR-CLIMATOR après un traitement statistique de correction de biais approprié.

\section{Résultats}

Afin d'avoir une idée à la fois statistiquement stable et précise du changement climatique sur la France, la différence entre deux normales climatiques entre le début et la fin de la simulation a été calculée. L'usage de moyennes trentenaires pour caractériser le climat a été respecté et nous considérons ici la différence entre les moyennes de la période 2071-2100 et celles de la période 1961-1990. Bien que le modèle soit global et donc que des valeurs sur le monde entier soient disponibles, nous nous concentrons sur la France métropolitaine. Lorsqu'on s'intéresse à la première moitié du $\mathrm{XXI}$ siècle, ce qui est légitime puisqu'elle nous concerne plus directement, l'analyse des résultats est polluée par la variabilité naturelle du climat, dont l'amplitude dépasse la réponse au forçage anthropique jusque vers 2030 pour la température et 2060 pour les précipitations. Aussi, il est plus pertinent de considérer que la réponse purement « effet de serre » de la première moitié du siècle est une fraction du signal que nous allons étudier dans ce qui suit. Une autre solution consisterait à produire plusieurs simulations de scénario identiques en termes de forçage mais indépendantes en termes de chronologie et à prendre leur moyenne.

La figure 1 montre la carte sur la France de la répartition de la réponse de la température hivernale pour les trois scénarios. Rappelons que le modèle ARPEGE fournit une réponse sur tous les points du globe, y compris les océans. Pour des raisons de lisibilité, nous avons masqué les points hors de notre domaine d'étude. Avec le scénario $\mathrm{A} 2$, la température augmente de $3{ }^{\circ} \mathrm{C}$ sur le Sud et de $2{ }^{\circ} \mathrm{C}$ sur le Nord. Avec le scénario $A 1 B$, le réchauffement n'est que de $2,5^{\circ} \mathrm{C}$ et le contraste Nord-Sud est remplacé par un contraste Est-Ouest, qui était déjà visible dans le scénario A2 sur la moitié Nord. On retrouve ce contraste Nord-Sud dans le scénario $B 1$, mais le réchauffement $n^{\prime}$ est plus que de $1,5^{\circ} \mathrm{C}$.

En été (figure 2) le réchauffement est plus important et la structure spatiale est en meilleur accord entre les trois scénarios. Le maximum a lieu sur le Sud-Ouest et le minimum sur les côtes de la Manche. Avec le scénario $A 2$, le réchauffement maximum est de $7{ }^{\circ} \mathrm{C}$ et le minimum est de $4{ }^{\circ} \mathrm{C}$. Avec le scénario $\mathrm{A} 1 \mathrm{~B}$, on obtient respectivement $5{ }^{\circ} \mathrm{C}$ et $3^{\circ} \mathrm{C}$. Avec le scénario $\mathrm{B} 1$, on obtient respectivement $3,5^{\circ} \mathrm{C}$ et $2^{\circ} \mathrm{C}$.

En ce qui concerne les précipitations, il est plus parlant de raisonner en terme de rapport plutôt qu'en terme de différence, car une diminution de $1 \mathrm{~mm} /$ jour n'aura pas les mêmes conséquences dans une région sèche et dans une région humide. La figure 3 montre la réponse pour l'hiver. Les trois scénarios s'accordent à proposer une diminution sur le Sud-Ouest et une augmentation sur le Sud-Est et, dans une moindre mesure, sur les pays de Loire. Dans le scénario A2, I'augmentation atteint $30 \%$ et la diminution atteint $20 \%$. Dans le scénario A1B, la diminution reste du même ordre, mais l'augmentation plafonne à $10 \%$. Dans le scénario B1 les deux effets atteignent à peine $10 \%$.

En été, comme on l'a vu plus haut pour la réponse thermique, la réponse hydrique est plus marquée qu'en hiver (figure 4). La diminution des précipitations concerne tout le pays, avec un contraste Est-Ouest dans tous les scénarios. Pour le scénario $A 2$, la réduction atteint $60 \%$ des précipitations sur la façade Atlantique, contre $20 \%$ sur le Sud des Alpes. Dans le scénario $A 1 B$, la réduction maximum est de $50 \%$ (Bretagne et Landes), tandis que la réduction de $20 \%$ s'étend au quart Sud-Est.
Avec le scénario $B 1$, ces réductions tombent à $40 \%$ et $10 \%$ respectivement.

\section{Conclusion}

Les résultats des scénarios étudiés ici montrent une élévation des températures surtout en été, une augmentation modérée des pluies hivernales sur quelques régions, et surtout une diminution des précipitations d'été jusqu'à $60 \%$. Nous n'avons pas mentionné les tempêtes et vents forts. Selon les études actuelles, l'augmentation du risque concernerait surtout le Nord de I'Europe. L'augmentation des vents extrêmes, calculée par des méthodes indirectes, sur le territoire métropolitain ne concernerait que les côtes de la Manche, et ce de manière marginale (quelques $\mathrm{m} / \mathrm{s}$ pour la valeur de retour centennale, pas d'impact sur la fréquence des événements).

Nous nous sommes limités ici aux scénarios $A 2$, $A 1 B$ et $B 1$ du GIEC. II en existe d'autres, intermédiaires (B2) ou extrêmes (A1Fl, scénario de stabilisation à $450 \mathrm{ppm}$ ). Le choix du forçage radiatif $n^{\prime}$ est pas le seul critère d'incertitude. L'utilisation d'autres jeux de TSM peut conduire à un réchauffement plus élevé avec notre modèle ARPEGE. L'utilisation d'autres modèles régionaux est mise en place dans le projet européen ENSEMBLES. Les résultats mis en avant ici ne sont pas remis en question par nos partenaires européens en ce qui concerne la réponse des températures (en toutes saisons) et des précipitations (en été). En ce qui concerne la réponse des précipitations d'hiver sur la France, certaines simulations montrent une diminution sur tout le pays, d'autres une augmentation sur la moitié nord. II convient de rester très prudent pour ce phénomène, à plus forte raison si on veut tirer des conclusions sur la première moitié du XXI ${ }^{\mathrm{e}}$ siècle.

Remerciements. Cette étude a été co-financée par la Commission européenne dans le cadre des projets FP6-ENSEMBLES (GOCE-CT-2003-505539) et FP6-CECILIA (COCE-CT-2006-037005) et par le projet ANR-CLIMATOR

\section{RÉFÉRENCES}

1. IPCC. In : Solomon S, Qin D, Manning M, Chen Z, Marquis M, Averyt KB, Tignor M, Miller HL, eds. Climate Change 2007 : the scientific basis. Contribution of Working Group I to the fourth assessment report of the Intergovernmental Panel on Climate Change. Cambridge, UK and New York, USA: Cambridge University Press, 2007. 
2. Cubasch U, Waszkewitz J, Hegerl G, Perlwitz J. Regional climate changes as simulated in timeslice experiments. Climatic Change 1995 ; 31 : 273-304.

3. Giorgi F, Mearns LO. Regional climate modeling revisited. An introduction to the special issue. / Geophys Res 1999 ; 104 : 6335-52.
4. Wilby RL, Wigley TML, Conway D, et al. Statistical downscaling of general circulation model output: A comparison of methods. Water resources research $1998 ; 34: 2995-3008$.

5. Déqué $M$, Piedelievre JP. High-Resolution climate simulation over Europe. Climate Dynamics $1995 ; 11: 321-39$.
6. Déqué M. Frequency of precipitation and temperature extremes over France in an anthropogenic scénario : model results and statistical correction according to observed values. Global and Planetary Change 2007 ; 57 : 16-26. 